\title{
Emission characteristics of photonic crystal light-emitting diodes
}

\author{
Ali Z. Khokhar, ${ }^{1}$ Keith Parsons, ${ }^{2}$ Graham Hubbard, ${ }^{2}$ Ian M. Watson, ${ }^{3}$ Faiz Rahman, ${ }^{1, *}$ \\ Douglas S. Macintyre, ${ }^{1}$ Chang Xiong, ${ }^{3}$ David Massoubre, ${ }^{3}$ Zheng Gong, ${ }^{3}$ Erdan Gu, ${ }^{3}$ \\ Nigel P. Johnson, ${ }^{1}$ Richard M. De La Rue, ${ }^{1}$ Martin D. Dawson, ${ }^{3}$ Steve J. Abbott, ${ }^{2}$ \\ Martin D. B. Charlton, ${ }^{4}$ and Martin Tillin ${ }^{5}$ \\ ${ }^{1}$ Department of Electronics and Electrical Engineering, University of Glasgow, Oakfield Avenue, \\ Rankine Building, Glasgow G12 8LT, United Kingdom \\ ${ }^{2}$ MacDermid Autotype Ltd, Grove Road, Wantage OX12 7BZ, United Kingdom \\ ${ }^{3}$ Institute of Photonics, University of Strathclyde, Wolfson Centre, 106 Rottenrow, Glasgow G4 ONW, United Kingdom \\ ${ }^{4}$ School of Electronics \& Computer Science, University of Southampton, Southampton SO17 1BJ, United Kingdom \\ ${ }^{5}$ Sharp Laboratories of Europe Ltd, Oxford Science Park, Oxford OX4 4GB, United Kingdom \\ ${ }^{*}$ Corresponding author: f.rahman @elec.gla.ac.uk
}

Received 10 December 2010; revised 7 April 2011; accepted 7 April 2011; posted 8 April 2011 (Doc. ID 139480); published 24 June 2011

\begin{abstract}
Experimentally measured optical properties of photonic crystal LEDs are reported here. Photonic crystal and photonic quasi-crystal structures were fabricated on GaN epilayer LED wafer material using both direct-write electron beam lithography and nanoimprint lithography. Some of these structures were processed to make finished LEDs. Both electroluminescence and photoluminescence measurements were performed on these structures. Devices were characterized for their current-voltage characteristics, emission spectra, far-field emission pattern, and angular emission pattern. These results are useful for fabricating photonic crystal LEDs and assessing their operational properties. () 2011 Optical Society of America

OCIS codes: $\quad 230.3670,350.4238,160.5298,220.4241$.
\end{abstract}

\section{Introduction}

LEDs have a come a long way from their initial commercial development in the early 1960s. In nearly half a century of development, they have evolved from feeble emitters of light into high-power and highly efficient light-emitting devices. Because of ongoing worldwide developments in LED technology, these devices are being increasingly used in new application areas. Mainstream space lighting and display illumination are only two pertinent examples. In order to compete with more established technologies, such as incandescent bulbs and fluorescent

0003-6935/11/193233-07\$15.00/0

(C) 2011 Optical Society of America tubes, LEDs need to become even more efficient and offer emission characteristics that cannot be matched by other older technologies [1-3]. The incorporation of photonic crystal (PhC) structures on LEDs in recent years has been a step in this direction $[\underline{4}, \underline{5}]$. These structures enable more light to be extracted from the confines of LED chips and at the same time offer an opportunity to tailor the angular emission profile to suit the application in hand [6-9]. In this context, $\mathrm{PhCs}$ are fabricated on the emitting faces of LEDs as a set of shallow etched holes, typically a few hundred nanometers in diameter and spaced a few hundred nanometers from each other. For this purpose, a variety of nanofabrication technologies have been demonstrated to date. These range from optical interference lithography [10] and 
direct-write electron beam lithography [11] to both hard and soft stamp nanoimprint lithography (NIL) [12-15], as well as other techniques $[16,17]$. Once the $\overline{\mathrm{PhC}}$ structure has been defined in a suitable resist, the pattern is transferred to the underlying semiconductor by a dry etch process. Properly performed, nanolithography followed by dry etching can generate high-quality $\mathrm{PhC}$ patterns on substrates ranging from small pieces to entire wafers. Burgeoning commercial interest in this technology has resulted in the ready availability of the tools and consumables required to carry out $\mathrm{PhC}$ fabrication on LED wafers. As a result, the cost of this additional process in LED manufacture has been continually falling-leading to its wider adoption by both specialized and diversified semiconductor manufacturers. The additional cost of imprinting LED wafers with $\mathrm{PhC}$ patterns is easily justified by the enhanced performance of $\mathrm{PhC}$ LEDs over plain LEDs. We have carried out a detailed survey of the various options available for producing PhC structures on GaN/InGaN blue LED wafers. This was done in order to better understand the advantages and disadvantages offered by different fabrication techniques and to see which technique could become the mainstay of commercial LED manufacturing operations. The results of our study on the nanofabrication of PhC LEDs has been published elsewhere [18]. Here we present our findings from measurements carried out on PhC LEDs fabricated by us. These results demonstrate how PhCs modify the simple Lambertian emission profile of LEDs into more complicated angular patterns dictated by the details of the PhC pattern.

\section{Photonic Crystal Designs for LEDs}

When it comes to LEDs, the term photonic crystal has to be used with some caution for two reasons. One is that PhCs for LEDs are essentially twodimensional (2D) structures, as they are formed as a plane of spaced holes with defined diameter and pitch. These structures, therefore, do not feature the full photonic bandgap as will be the case for a three-dimensional photonic crystal structure. The other reason for exercising caution is that the $\mathrm{PhC}$ holes are quite shallow, so that the structure only acts as a weak diffraction grating. This has to be so by necessity because deeper holes will penetrate inside the light-generating quantum well region of the LED, shorting the junction and severely impairing the light output of the device. The shallow $2 \mathrm{D}$ set of holes, nevertheless, is sufficient to usefully modify the emission characteristics of the LEDs that it is patterned on. Our work showed that as the etch depth of PhC holes increases, the coupling of trapped modes to the $\mathrm{PhC}$ also increases, but on reaching the quantum well structure (usually there are five GaN/ InGaN quantum wells in typical commercial LEDs), the LEDs show an abrupt rise in leakage current, and their light emission quickly deteriorates. As a safe rule, we recommend that $\mathrm{PhC}$ holes be etched down to $80 \%$ of the distance from the surface to the top of the quantum well structure. This depth, however, has to be taken into account during the $\mathrm{PhC}$ design process, as etching to different depths can also modify the angular radiation pattern from PhC LEDs. In this work, the photonic crystal holes were kept deliberately shallow due to secondary (hard) mask erosion considerations. The etch terminated $100 \mathrm{~nm}$ above the multi quantum well layer, which was located $200 \mathrm{~nm}$ below the surface of the wafer. This limited the possible intensity enhancement, but with better resists and hard masks, deeper holes can be achieved, and thus significantly larger gains in intensity are possible.

$\mathrm{PhCs}$ can be regular periodic or quasi-periodic. Regular periodic PhCs belong to one of the Bravais lattices in two dimensions. Square and triangular symmetry regular lattices are most common. Quasiperiodic photonic crystals possess no short-range order but have long-range periodicity. We used both types in our investigations but with more emphasis on the use of quasi-periodic structures. Our regular periodicity $\mathrm{PhC}$ design was formed from the tiling of hexagonal symmetry dot patterns, whereas the quasi-periodic design was based on a twelvefold symmetry pattern. In addition, we also made use of a quasi-random $\mathrm{PhC}$ design obtained from a distorted square lattice through periodic tiling. Our regular and quasi-periodic designs utilized $200 \mathrm{~nm}$ designed hole diameters spaced on a $400 \mathrm{~nm}$ pitch and etched down to typically $100 \mathrm{~nm}$ into the LED wafer. The quasi-random design had a $200 \mathrm{~nm}$ design hole diameter with interhole distances varying from 200 to $500 \mathrm{~nm}$. The holes were etched down to about $100 \mathrm{~nm}$ in this case as well.

\section{Device Fabrication and Electrical Measurements}

$\mathrm{PhC}$ structures were fabricated on $\mathrm{GaN}$ epilayer LED wafer material using both direct-write electron beam lithography and nanoimprint lithography. After carrying out patterning on many wafers, we reached the conclusion that although electron beam lithography is expensive, it can be used for both research devices and for niche commercial devices where high performance is required, as in scientific applications such as illumination in near-field microscopy. NIL can produce yields in the range of $65 \%$ to $92 \%$ depending on such factors as incoming epitaxial
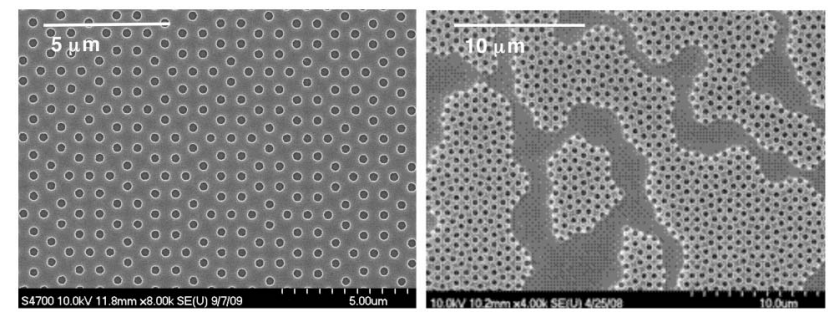

Fig. 1. Left, closeup scanning electron micrograph of a photonic quasicrystal pattern on a GaN LED chip. Right, closeup of defective patterning when the incoming wafer is not perfectly flat, leading to nonuniform nanoimprinting. 


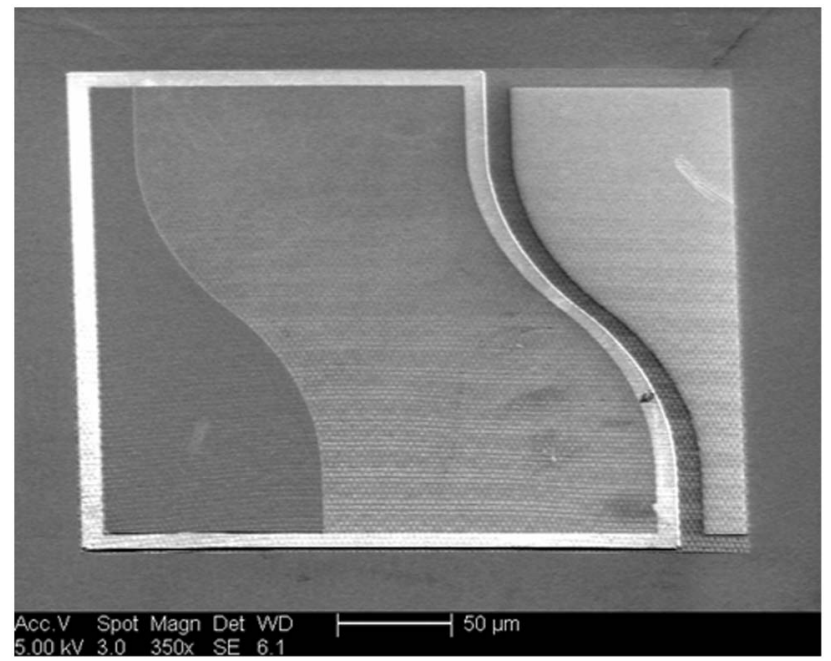

Fig. 2. SEM micrograph of one of the PhQC LEDs showing (left) $p$ contact and (right) $n$ contact.

wafer quality (wafer bow and particulate contamination being the main factors limiting production yield) and the quality of the imprinting stamp. Figure 1 shows scanning electron microscope (SEM) images of a photonic quasi-crystal ( $\mathrm{PhQC}$ ) pattern on one of our LEDs. The image on the left is from a good device, whereas the image on the right shows a defective pattern as a result of the departure of the wafer from flatness. After PhC patterning, dry etch-resistant resists were employed for pattern transfer onto the semiconductor through an inductively coupled plasma dry etch process. LED structures measuring $350 \mu \mathrm{m} \times 350 \mu \mathrm{m}$ were fabricated using standard photolithography, dry etching, and metallization techniques. An SEM micrograph of our finished PhQC LED device appears in Fig. 2. The $n$ contact pad, seen to the right, required etching down to the $n$-type contact layer of the epitaxial structure, whereas the $p$ contact pad was formed directly on top of the wafer. A thin Ni/Au bilayer current spreading metal was deposited on the top face of the LED to distribute current evenly across the entire cross section of the device. This transparent conductive layer is needed in all large area LEDs, because the relatively low electrical conductivity of $p$-type GaN would otherwise lead to current crowding effects, resulting in uneven illumination from the emitting face of the LED. In order to further reduce current crowding effects and the resulting thermal nonuniformity effects in the LED chip, we utilized anode and cathode electrode designs developed by Bulashevich et al. from the simulation of such effects in blue GaN LEDs [19]. The curved contours of this design are clearly seen in Fig. 2, which shows one LED die. We tested our devices both on-wafer and after dicing and bonding to TO-style headers. Their IV characteristics, shown in Fig. $\underline{3}$, are essentially identical to unpatterned reference devices made on the same chips, thus showing that the $\mathrm{PhQC}$ patterning process does not cause electrical degradation of LEDs. Furthermore, the IV curves are tightly bunched together, showing small variability of characteristics from device to device. Such uniformity in the currentvoltage characteristic is remarkable, as most current commercial offerings of unbinned LED devices show much higher deviations in device characteristics. The turn-on voltage was $3.5 \mathrm{~V} \pm 0.1 \mathrm{~V}$, with bright blue emission obtained at $4 \mathrm{~V}$. These voltages are better

\section{Lumi Innovations wafer D5719165, 5 devices, PhQC depth $100 \mathrm{~nm}$}

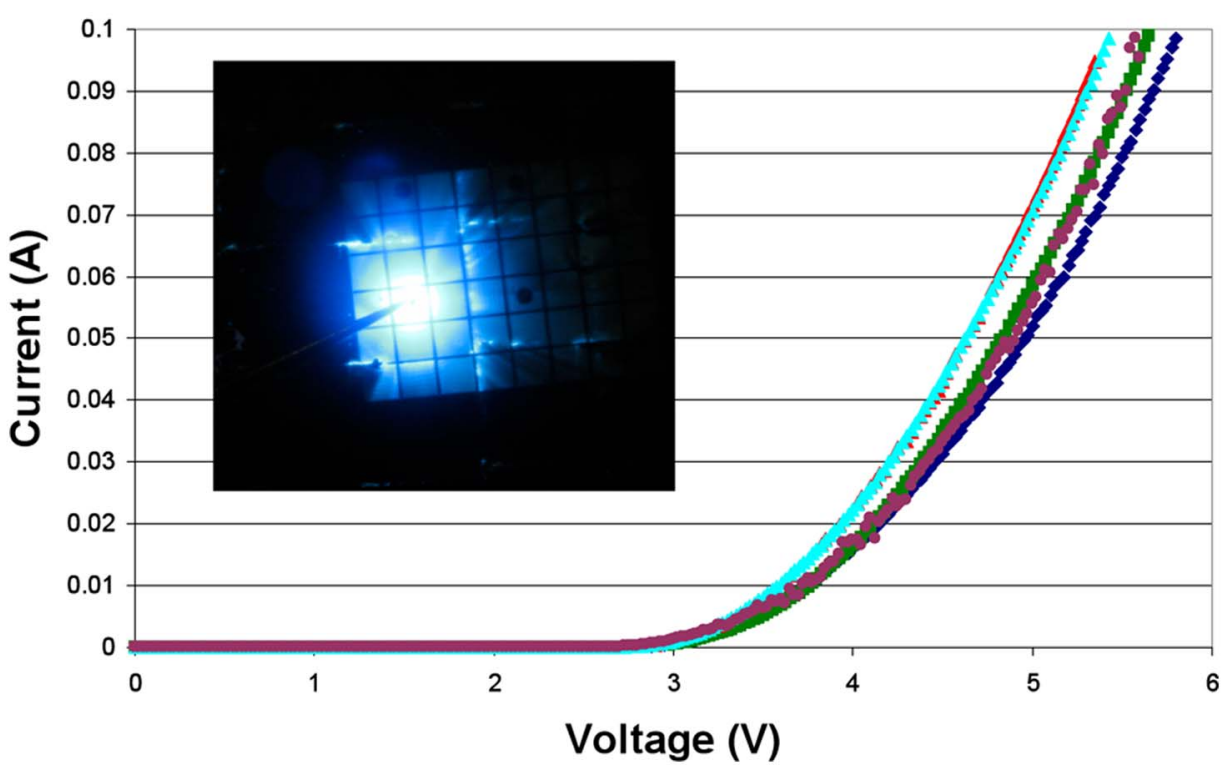

Fig. 3. (Color online) Current-voltage characteristics of several PhQC LEDs with $100 \mathrm{~nm}$ hole depth. Inset shows light emission from one LED during on-wafer testing. 

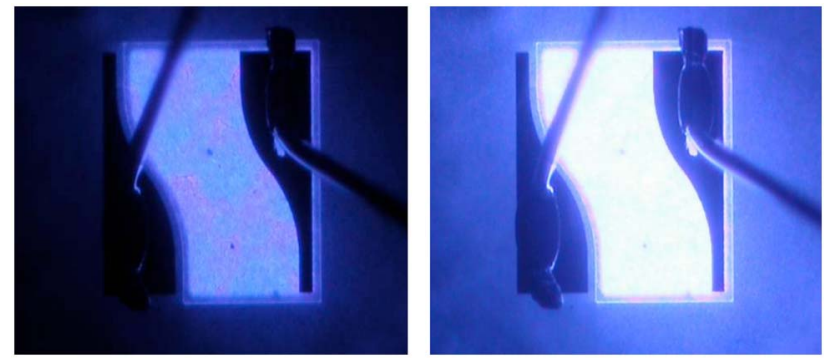

Fig. 4. (Color online) Light emission from PhQC LED. Left, low current operation. Right, high current operation.

than those of many commercially available blue LEDs and attest to the high quality of the electrical contacts achieved in our devices. The low forward voltage drop, small spread in voltage drops, and almost identical IV characteristics measured for more than two dozen devices attests to the usefulness of the particular electrode design used in our devices. We therefore recommend that this electrode design be used for commercial GaN LEDs. These LEDs performed very well in our tests, generating strong and uniform emission from the top emitting face. Figure 4 shows an operating PhQC LED. The figure on the left was taken with the device operating at $1 \mathrm{~mA}$ injection current, whereas the figure on the right was taken with the device operating at $3 \mathrm{~mA}$ injection current. The uniformity of light emission over the entire light-emitting region of the device is remarkable and attests to the high quality of our fabrication process. We believe that proper spreading metal deposition as well as the choice of our electrode design were factors that led to the excellent emission uniformity. Further details on device fabrication can be found in our earlier publication related to the fabrication of these devices [18].

\section{Optical Measurements}

Characterization of the light emission pattern of $\mathrm{PhC}$ LEDs through their electroluminescence (EL) was

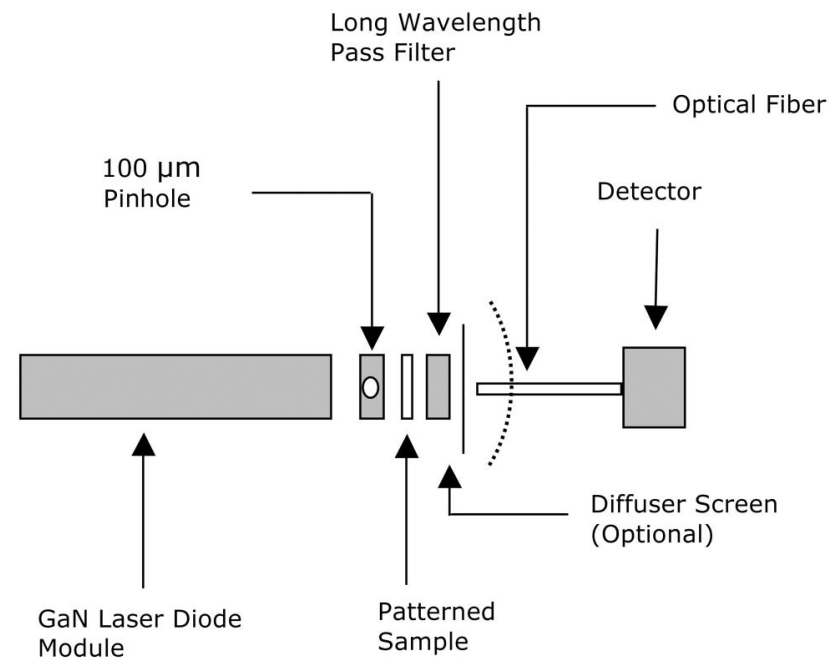

Fig. 5. Experimental setup for exciting and measuring angleresolved photoluminescence from patterned GaN LED material samples. The dotted curve shows the probe track. carried out on complete LED devices with quasiperiodic $\mathrm{PhC}$ designs. In addition, all three designsregular, quasi-periodic and quasi-random-were also characterized by measuring the angular distribution of their photoluminescence (PL) using a customized setup shown in Fig. 5. This consisted of a $405 \mathrm{~nm}$ GaN/InGaN blue laser diode module operating at $9 \mathrm{~V}$ nominal operating voltage, used as a pump light source. This was followed by a $100 \mu \mathrm{m}$ circular pinhole aperture for selectively illuminating desired areas on patterned LED wafer pieces. The sample was followed by a long-pass filter capable of rejecting wavelengths shorter than $430 \mathrm{~nm}$. This filter ensured that no residual pump light could reach the detector and impair its functionality. An optional white diffuser screen could be placed beyond the filter, if needed, to observe the illumination pattern. An optical fiber with a circular $100 \mu \mathrm{m}$ aperture was used to receive the light from the sample and take it to a calibrated silicon detector. The optical entrance end of the optical fiber was mounted on a semicircular track goniometer so that light at different angular positions from the normal to the sample could be observed. It should be mentioned here that the technique described above is, to our knowledge, novel as far as its application to surface-structured LEDs is concerned. Although PL characterization is routinely used by epitaxial wafer growers, ours is, to our knowledge, the first description of this setup for quick characterization of the efficacy of $\mathrm{PhC}$ structures on LEDs.

On illuminating any patterned area on our sample, we could observe a corresponding laser light diffraction pattern. This pattern is the Fourier transform of the pattern that was physically etched on the semiconductor material. These diffraction patterns for all three experimental patterns are depicted here in Fig. 6. The sixfold symmetry hexagonal $\mathrm{PhC}$ structure produced the pattern on the left. Notice the fainter secondary spots that accompany the six secondary maxima. These indicate the presence of an additional periodicity in pattern placement that arises due to field stitching errors in electron beam lithography when pattern fields are tiled together to cover the entire substrate. In this way, diffraction images reveal any irregularities in the $\mathrm{PhC}$ structure. The image in the middle is the diffraction pattern from our twelvefold symmetry quasi-periodic
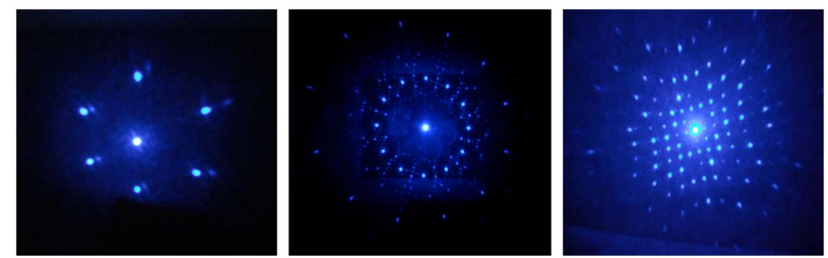

Fig. 6. (Color online) Diffraction images generated when a $405 \mathrm{~nm}$ pump laser beam interacts with the photonic crystal pattern on an LED wafer. Left, diffraction from regular sixfold symmetry $\mathrm{PhC}$ pattern. Middle, from a twelvefold symmetry $\mathrm{PhQC}$ pattern. Right, from a quasi-random $\mathrm{PhC}$ pattern. 


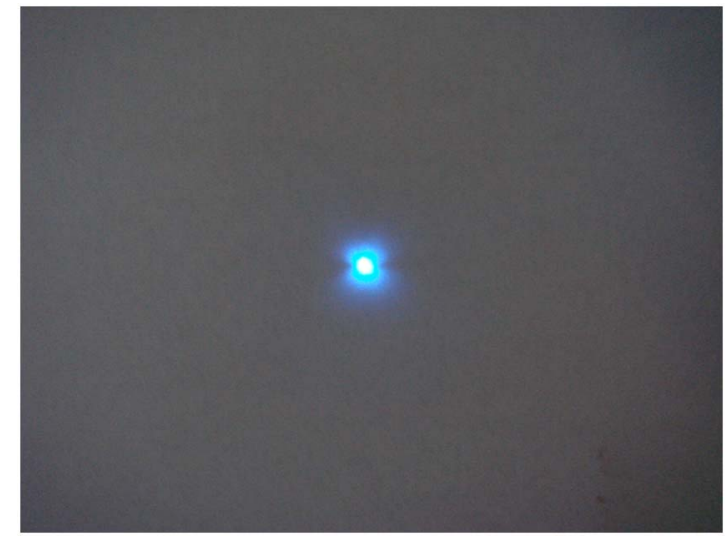

Fig. 7. (Color online) Far-field radiation pattern from a photonic quasi-crystal LED chip operated with $8 \mathrm{~mA}$ of forward current. The pointed dark protruding features at the right and left of the center are due to bond wires.

structure and clearly shows many other (tertiary) diffraction spots that arise from the quasi-periodicity of this structure. Finally, the distorted quasi-random square structure produced the pattern that appears on the right of this figure. Notice here that, because our quasi-random pattern was obtained by modifying a square lattice, the underlying symmetry is still evident in the diffraction pattern; the pincushion distortion in this case is the contribution to the diffraction pattern from the quasi-random placement of $\mathrm{PhC}$ holes in this case. In all cases, the undiffracted central maxima are also seen. These patterns could be imaged either using either a diffuser screen and camera or a direct CCD imaging technique whose details will be published later. The former method was used for the images that appear in Fig. 5. The diffraction patterns were seen to disappear completely
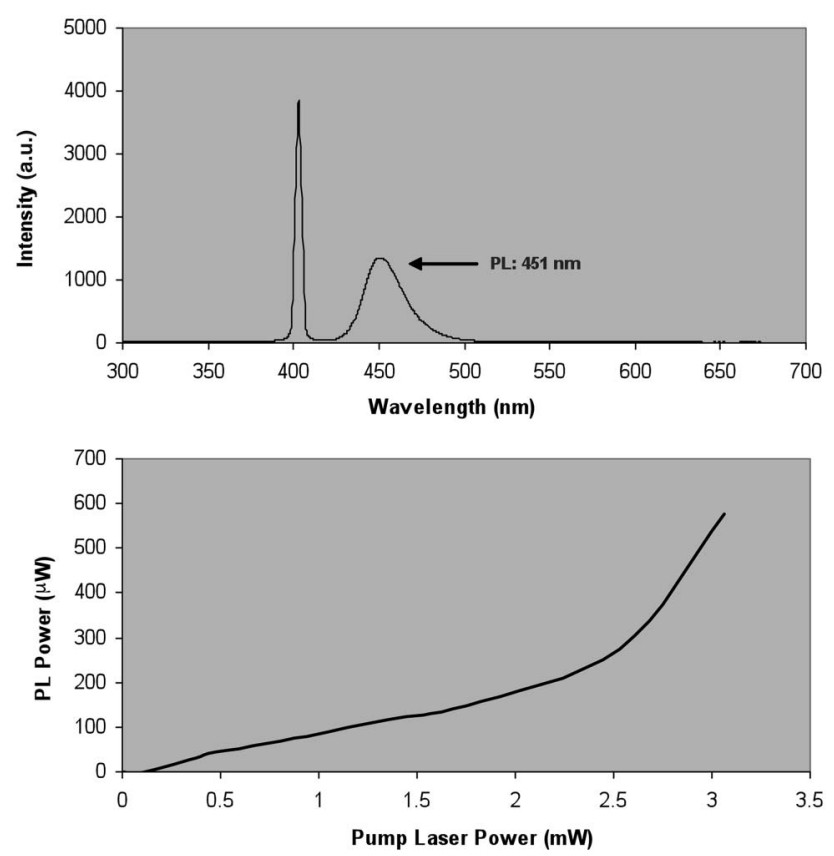

Fig. 8. Spectral plot showing the pump laser beam at $405 \mathrm{~nm}$ and the PL emission from the patterned LED wafer material at $451 \mathrm{~nm}$. when the long-pass filter was introduced after the sample. This served as a test to ensure that only the LED PL reached the photodetector during later measurements. The far-field EL of these LEDs is quite featureless, as seen in Fig. 7. No evidence of diffraction spots is seen, because in this case light is uniformly generated all over the LED surface and couples collectively to the PhQC structure. The outline of the chip is also seen in this image, together with light that spilled out of the sapphire substrate. These were the laterally propagating sapphire modes that cannot be extracted through $\mathrm{PhC}$ patterns in the epitaxial layers above.

The upper panel in Fig. 8 is a visible light spectrum that shows both the pump laser radiation at $405 \mathrm{~nm}$ and the resulting LED PL at $\sim 451 \mathrm{~nm}$. The lower panel shows the dependence of PL intensity on the intensity of pump light. This measurement was taken from an unpatterned location on the sample and is similar to subsequent measurements taken from patterned areas. It is interesting to note that the PL intensity shows a marked increase at pump powers in excess of $2.5 \mathrm{~mW}$.

Measurements of angle-resolved PL from the three types of $\mathrm{PhC}$ structures are compared with the emission from an unpatterned area in Fig. 9. The plots show the average response from both sides of the normal. A featureless location on the GaN LED wafer showed angular emission that was narrow in the center, but broadened after about $4^{\circ}$ from the normal to the device. The quasi-periodic $\mathrm{PhC}$ structure location yielded a similar distribution but with higher intensity normal to the device plane, thus indicating that such a structure would be useful for making pointsource emitters. However, on changing the PhC hole depth to $200 \mathrm{~nm}$, small secondary peaks appeared, which are seen in Fig. 10. This figure also shows a comparison between the angular emission as measured through EL and PL emissions. The agreement between the two is quite good; giving us confidence

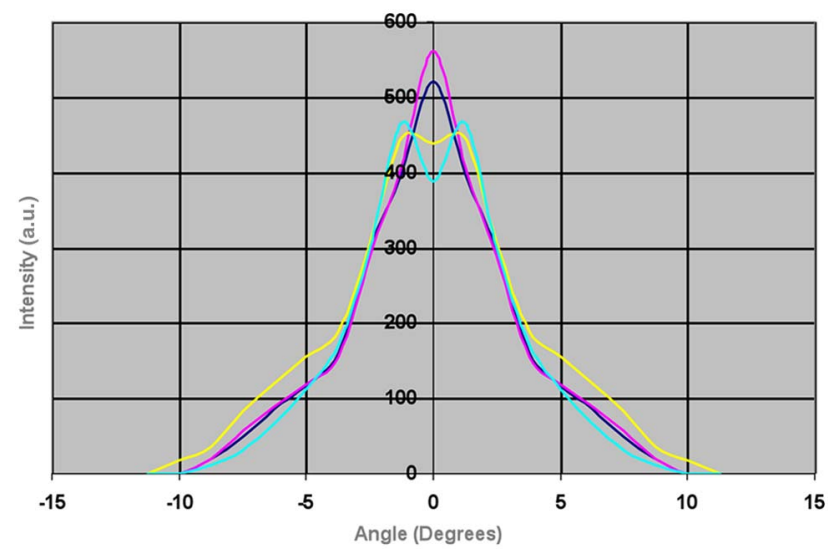

Fig. 9. (Color online) Angle-resolved PL intensity profiles for one unpatterned and three patterned GaN LED samples. The patterns tested were: regular hexagonal photonic crystal (RPC), 12-fold symmetry quasi-periodic photonic crystal (QPC) and a square symmetry quasi-random photonic crystal. All patterns were etched down to a depth of $100 \mathrm{~nm}$ into the wafer material. 


\section{PL-EL comparison}

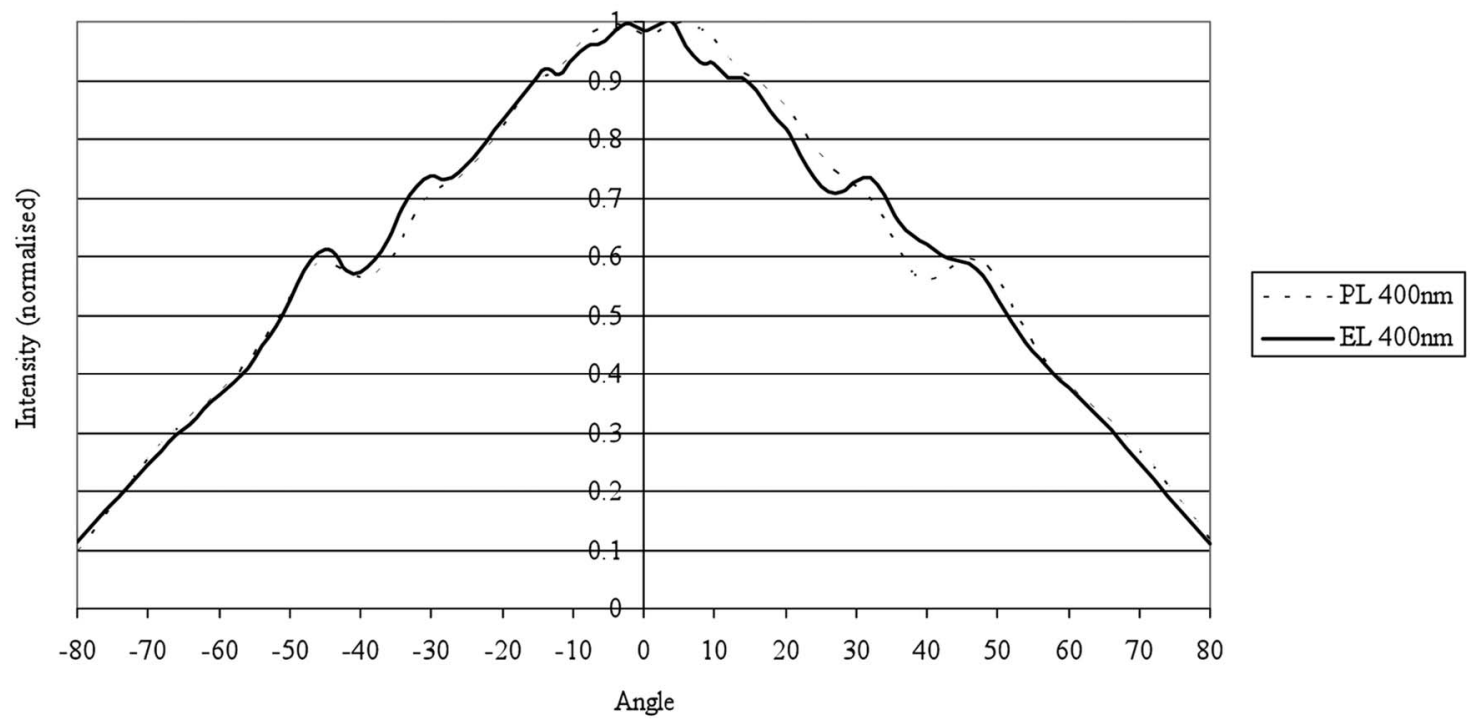

Fig. 10. Comparison of the intensity profiles as recorded through electroluminescence (EL) and photoluminescence (PL) measurements.

that PL measurements yield results that are representative of finished LED devices emitting light with current injection. The angular emission patterns of regular $\mathrm{PhC}$ and quasi-random $\mathrm{PhC}$ structures shown in Fig. 9 are interesting as both show reduced intensity normal to the device and well-defined side lobes. Also notable is the fact that, while shallowetched quasi-periodic $\mathrm{PhC}$ structures did not show evidence of side lobes or major modification of their angular emission pattern, these structures did show enhanced light extraction capability. This can be clearly seen from Fig. 9, where results have not been normalized and can thus be compared against each other. Such enhancement of light output through extraction of trapped modes is now well documented and has been reported by several groups [20-25]. Shallow-etched quasi-periodic $\mathrm{PhC}$ structures thus appear well-suited to enhancing the brightness of GaN LEDs, whereas other structures could be used for broadening the light emission pattern for applications such as LCD backlight displays. Deeper quasi-periodic $\mathrm{PhC}$ structures can alter the emission profile substantially and indeed, our LEDs with deeper structures show clear evidence of that, as seen in Fig. 8. Furthermore, by altering the size and spacing of $\mathrm{PhC}$ holes, it is possible to alter the position and extent of side lobes as we and others have reported previously [26-29].

\section{Conclusions}

We have fabricated and characterized blue-emitting GaN LEDs with PhC structures of various geometries. Regular hexagonal, twelvefold quasi-periodic and quasi-random designs were produced through both electron beam lithography and NIL followed by dry etching. The fabricated structures appeared very uniform, with vertical side walls and well-defined circular contours. Some of the quasi-periodic struc- tures were made into complete operating LEDs and were also measured for their electrical performance. The devices appeared extremely homogenous. Measurement of angle-resolved EL and PL showed that shallow-etched $(\leq 100 \mathrm{~nm}) \mathrm{PhC}$ regions with quasi-periodic structures yield enhanced light output normal to the device. Quasi-random and regular $\mathrm{PhC}$ designs both showed reduced output normal to the device, with significant side lobes. These results provide a baseline guide for selecting appropriate structures for the functionality desired from blue-emitting GaN LEDs.

This work was supported by the UK Technology Strategy Board (TSB) under grant TP/6/EPH/6/S/ K25067 with the project title "Photonic Quasicrystal LEDs for Display Illumination (PQLDI)". Their support is gratefully acknowledged.

\section{References}

1. M. R. Krames, O. B. Shchekin, R. Mueller-Mach, G. O. Mueller, L. Zhou, and M. G. Craford, "Status and future of high-power light-emitting diodes for solid-state lighting," J. Display Technol. 3, 160-174 (2007).

2. K. McGroddy, A. David, E. Matioli, M. Iza, S. Nakamura, S. DenBaars, J. S. Speck, C. Weisbuch, and E. L. Hu, "Directional emission control and increased light extraction in GaN photonic crystal light-emitting diodes," Appl. Phys. Lett. 93, 103502-103502-3 (2008).

3. C.-F. Lai, J.-Y. Chi, H.-C. Kuo, C.-H. Chao, H.-T. Hsueh, J.-F. T. Wang, and W.-Y. Yeh, "Anisotropy of light extraction from GaN two-dimensional photonic crystals," Opt. Express 16, 7285-7294 (2008).

4. F. Rahman and A. Khokhar, "Holey LEDs target TV backlights," Comp. Semiconduc. 14, 23-25 (2008).

5. F. Rahman, "Photonic crystal LEDs," Opt. Photon. News 20(6), 24-29 (2009).

6. C. Weisman, K. Bergenek, N. Linder, and U. T. Schwarz, "Photonic crystal LEDs-designing light extraction," Laser Photon. Rev. 3, 262-286 (2009). 
7. T. N. Oder, J. Shakya, J. Y. Lin, and H. X. Jiang, "III-nitride photonic crystals," Appl. Phys. Lett. 83, 1231-1233 (2003).

8. A. David, T. Fujii, B. Moran, S. Nakamura, S. P. DenBaars, and C. Weisbuch, "Photonic crystal laser lift-off GaN lightemitting diodes," Appl. Phys. Lett. 88, 133514 (2006).

9. A. J. Fischer, G. Subramania, Y. J. Lee, T. S. Luk, J. R. Wendt, and D. D. Koleske, "Improved InGaN LED efficiency using photonic crystal patterning and surface plasmon-enhanced emission," ECS Trans. 13 (3), 5-11 (2008).

10. L. Pang, W. Nakagawa, and Y. Fainman, "Fabrication of two-dimensional photonic crystals with controlled defects by use of multiple exposures and direct write," Appl. Opt. 42, 5450-5456 (2003).

11. R. Steingrüber, S. Golka, and H. Heidrich, "Useful and costefficient fabrication of dot arrays for photonic crystals by direct-write electron-beam lithography," Microelectron. Eng. 67-68, 157-161 (2003).

12. E. M. Arakcheeva, E. M. Tanklevskaya, S. I. Nesterov, M. V. Maksimov, S. A. Gurevich, J. Seekamp, and C. M. Sotomayor-Torres, "Fabrication of semiconductor-and polymer-based photonic crystals using nanoimprint lithography," Tech. Phys. 50, 1043-1047 (2005).

13. S. H. Kim, K.-D. Lee, J.-Y. Kim, M.-K. Kwon, and S.-J. Park, "Fabrication of photonic crystal structures on light-emitting diodes by nanoimprint lithography," Nanotechnology 18, 055306-055310 (2007).

14. K.-J. Byeon, S.-Y. Hwang, and H. Lee, "Fabrication of twodimensional photonic crystal patterns on GaN-based lightemitting diodes using thermally curable monomer-based nanoimprint lithography," Appl. Phys. Lett. 91, 091106091108 (2007).

15. K.-J. Byeon, S.-Y. Hwang, C.-H. Hong, J. H. Baek, and H. Lee, "Insertion of two-dimensional photonic crystal pattern on $p$ GaN layer of GaN-based light-emitting diodes using bi-layer nanoimprint lithography," J. Nanosci. Nanotechnol. 8, 5242-5246 (2008).

16. W. N. Ng, C. H. Leung, P. T. Lai, and H. W. Choi, "Photonic crystal light-emitting diodes fabricated by microsphere lithography," Nanotechnology 19, 255302-255306 (2008).

17. W. Y. Fu, K. K.-Y. Wong, and H. W. Choi, "A novel GaN photonic crystal structure comprising nanopillars with inclined sidewalls," Phys. Status Solidi C 6, S639-S642 (2009).

18. A. Z. Khokhar, K. Parsons, G. Hubbard, F. Rahman, D. S. MacIntyre, C. Xiong, D. Massoubre, Z. Gong, N. P. Johnson, R. M. De La Rue, I. M. Watson, E. Gu, M. D. Dawson, S. J. Abbott, M. D. B. Charlton, and M. Tillin, "Nanofabrication of gallium nitride photonic crystal light-emitting diodes," Microelectron. Eng. 87, 2200-2207 (2010).
19. K. A. Bulashevich, I. Y. Evstratov, V. F. Mymrin, and S. Y. Karpov, "Current spreading and thermal effects in blue LED dice," Phys. Status Solidi C 4, 45-48 (2007).

20. H. K. Cho, J. Jang, J.-H. Choi, J. Choi, J. Kim, J. S. Lee, B. Lee, Y. H. Choe, K.-D. Lee, S. H. Kim, K. Lee, S.-K. Kim, and Y.-H. Lee, "Light extraction enhancement from nanoimprinted photonic crystal GaN-based blue light-emitting diodes," Opt. Express 14, 8654-8660 (2006).

21. J. Shakya, K. H. Kim, J. Y. Lin, and H. X. Jiang, "Enhanced light extraction in III-nitride ultraviolet photonic crystal light-emitting diodes," Appl. Phys. Lett. 85, 142-144 (2004).

22. H. W. Huang, C. H. Lin, K. Y. Lee, C. C. Yu, J. K. Huang, B. D. Lee, H. C. Kuo, K. M. Leung, and S. C. Wang, "Enhanced light output power of GaN-based vertical-injection lightemitting diodes with a 12 -fold photonic quasi-crystal by nanoimprint lithography," Semicond. Sci. Technol. 24, 085008 (2009).

23. A. A. Erchak, D. J. Ripin, S. Fan, P. Rakich, J. D. Joannopoulos, E. P. Ippen, G. S. Petrich, and L. A. Kolodziejski, "Enhanced coupling to vertical radiation using a two-dimensional photonic crystal in a semiconductor light-emitting diode," Appl. Phys. Lett. 78, 563-566 (2001).

24. J. J. Wierer, Jr., A. David, and M. M. Megens, "III-nitride photonic crystal light-emitting diodes with high extraction efficiency," Nat. Photon. 3, 163-169 (2009).

25. P. A. Shields, M. D. B. Charlton, T. Lee, M. E. Zoorob, D, W. E. Allsopp, and W. N. Wang, "Enhanced light extraction by photonic quasi-crystals in GaN blue LEDs," IEEE J. Sel. Top. Quantum Electron. 15, 1269-1274 (2009).

26. M. Tillin, M. D. B. Charlton, Z. Gong, A. Z. Khokhar, D. Massoubre, I. M. Watson, E. Gu, M. D. Dawson, F. Rahman, N. P. Johnson, D. Mcintyre, R. M. De La Rue, K. Parsons, and S. Lin, "Photonic quasi-crystal light-emitting diodes: comparisons of device performance with pattern pitch," Proc. SPIE 7713, 77130B (2010).

27. K. Kim, J. Choi, J. B. Park, S. C. Jeon, J. S. Kim, and H. M. Lee, "Lattice constant effect of photonic crystals on the light output of blue light-emitting diodes," IEEE Photon. Technol. Lett. 20, 1455-1457 (2008).

28. M. Rattier, T. F. Krauss, J. F. Carlin, R. Stanley, U. Oesterle, R. Houdre, C. J. M. Smith, R. M. De La Rue, H. Benisty, and C. Weisbuch, "High extraction efficiency, laterally injected, light-emitting diodes combining microcavities and photonic crystals," Opt. Quantum Electron. 34, 79-89 (2002).

29. A. David, T. Fujii, R. Sharma, K. McGroddy, S. Nakamura, S. P. DenBaars, E. L. Hu, C. Weisbuch, and H. Benisty, "Photonic crystal GaN light-emitting diodes with tailored guided modes distribution," Appl. Phys. Lett. 88, 061124-061126 (2006). 